УДК 621.396 .967

DOI: 10.15827/0236-235X.133.019-032

\title{
Ментальные модели в проектировании поведения искусственнъх сущностей
}

\author{
Г.П. Виноградов 1,2, д.m.н., профессор, зав. лабораторией, wgp272ng@mail.ru
}

\author{
1 Тверской государственный технический университет, \\ 2. Тверь, 170026, Россия \\ 2 НИИ "Центрпрограмисистем", г. Тверь, 170024, Россия
}

Актуальность рассматриваемой в данной статье проблемы связана с необходимостью проектирования искусственных систем для выполнения некоторой миссии подобно человеку и при взаимодействии с ним. Анализ подходов к построению искусственных сущностей показал, что разработчики зачастую наделяют их своими паттернами поведения в силу специфики используемых ими понятий и концепций. В результате возникают разрывы между формальными моделями паттернов, предлагаемых разработчиками, и ожиданиями со стороны пользователей.

Цель авторов статьи - обосновать подход к разработке ПО интеллектуальных систем управления поведением искусственных сущностей на базе теории паттернов, обеспечивающий разработку цифровых продуктов на основе паттернов поведения субъекта-лидера для систем управления искусственными сущностями, автоматизирующих выполнение задач миссии.

В работе использованы методы теорий рефлексивных игр и информационного управления системами, обладающими волей и интеллектом. На основе формальной модели паттерна поведения выделены десять составных элементов. Показано, что эти элементы образуют то, что можно назвать интеллектуальной цифровой машиной, предназначенной для автоматизации выполнения миссии в интересах субъекта-хозяина. Эти составные элементы присутствуют при выполнении миссии человеком, в том числе и у всех участников проекта по разработке искусственной сущности. Показано, что согласование представлений об архитектуре искусственной сущности путем обмена информацией при обсуждении позволяет определить наиболее эффективную модель паттерна поведения и всех ее составляющих для реализации в искусственной сущности. Согласование предложено проводить в форме игры путем проведения экспериментов по методу ТОТЕ (набор входных данных $\rightarrow$ воздействие $\rightarrow$ результат), используя пространственную и визуальную логику для интерпретации результатов. Обоснована возможная логика интерпретации. Кратко рассмотрены модели составных элементов паттерна поведения искусственной сущности, а также пример реализации подхода. Показано, что в условиях жесткого дефицита времени выбор на основе паттернов поведения позволяет реализовать эффективное поведение, не требующее значительных вычислительных ресурсов.

Ключевые слова: принятие решений, целеустремленные системы, нечеткое суждение, паттерн, ситуация выбора.

Интерес к проблеме построения интеллектуальных машин связан с задачами управления их поведением в организационно-технических системах [1-4]. Основным средством для решения подобного рода задач является агентноориентированное моделирование $[1,2]$. Согласно этому подходу, искусственная сущность (далее - агент) обладает способностью взаимодействовать с внешней средой, но не может ее контролировать.

Поведение искусственных сущностей на основе формальных моделей исследуется в теориях агентов, принятия решений, поведенческой робототехники, искусственного интеллекта и в ряде других направлений [5-7]. Цель исследований - разработка автономной искус- ственной сущности, демонстрирующей интеллектуальное поведение, связанное с рассуждениями и принятием решений.

В классической BDI-архитектуре агента рассуждения выполняются с использованием механизмов логического вывода на основе ментальных понятий, представленных некоторыми структурами знаний [6]. Однако реализация логического вывода требует больших затрат вычислительных ресурсов и времени при планировании поведения, к тому же приводит к возникновению проблемы актуализации и выбора стратегии [8-11].

Формальная теория выбора [11] развивалась путем абстрагирования от субъективных факторов, что привело к созданию нормативной 
теории принятия решений идеальным субъектом. Ограничения, накладываемые общей логической и математической абстракцией классической теории принятия решений на процедуры принятия решений, практически сводят науку о решениях к совокупности механических приемов. Сущности рассматриваются как объекты, механически реагирующие на входные стимулы, что исключает возможность управления выбором.

Успешно моделировать поведение можно только тогда, когда оно будет определено понятиями (переменными), значения которых можно получить, воспринимая регулярность поведения в сходных ситуациях. Тогда моделирование поведения становится возможным на основе вывода функциональных свойств из наблюдаемого поведения, что позволяет воспроизводить его в цифровых машинах. В работе [12] предложены идеализированные конструктивные определения и способы измерения таких функциональных понятий на основе положений теории вероятностей и экспертных оценок, психологии, социологии и системного анализа. Дальнейшее развитие этого подхода на базе теории нечетких множеств было связано с обоснованием понятия субъективно рационального выбора, зависящего от субъективных представлений о ситуации выбора лица, принимающего решение [13-15]. Там же было показано, что в условиях ограниченных ресурсов мозг использует энергосберегающие и когнитивные технологии в форме доступных паттернов. В силу ограниченных вычислительных возможностей мозга по хранению и обработке информации паттерны имеют достаточно простую структуру [16].

По сути паттерны - это ментальные модели поведения естественных сущностей (субъектов) в предметной области. Они основаны как на фактах, так и на убеждениях, когда сущность знает (или думает, что знает) о процессах конкретной предметной области. Каждый отдельный субъект является носителем своего индивидуального паттерна поведения. Они могут быть и схожими, и разными, то есть субъекты могут создавать различные модели поведения (паттерны) в одних и тех же ситуациях. Ментальная модель - это процесс мышления, необходимый для придания смысла жизненному опыту, выявления причин и следствий и формирования убеждений для объяснения того, как что-то работает. Разработчики, например, наделяют искусственные сущности своими паттернами поведения в силу специфики используемых ими понятий и концепций. Так они реализуют свои убеждения. В результате возникают разрывы между формальными моделями паттернов, предлагаемых разработчиками, и ожиданиями со стороны пользователей.

В связи с этим актуальна проблема построения моделей паттернов поведения, которые отражают реальные аналоги, имеющие наибольшую эффективность. Поэтому решение проблемы построения ПО интеллектуального поведения искусственной сущности должно быть основано на изучении и моделировании поведения субъектов-лидеров. Цель - выявление и описание их ментальных моделей в реальной предметной области, а также используемых ими способов повышения эффективности своего поведения.

\section{Субъективно рациональный выбор}

Развитие идеи субъективно рационального выбора открыло возможности объяснения принятия решений субъектом в конкретных ситуациях, предсказания возможных реакций искусственной сущности в различных ситуациях, решения задач активного прогноза, когда управляющая сторона создает у управляемой нужный образ будущего [16, 17].

Субъективно рациональный выбор предполагает, что наиболее сильное влияние на результат оказывают мотивация субъекта и его представления о ситуации выбора, которые он рассматривает как модель. Мотивация определяется как внешними, так и внутренними факторами. Внутренние факторы отражают интересы субъекта, индуцируемые его потребностями и этической системой, которой он придерживается. Модель ситуации выбора строится субъектом на основе информации, получаемой им через органы чувств из окружения, от других субъектов и от самого себя (опыт). Основную роль играют три процесса: восприятие, осознание, понимание. Субъективную информацию человек рассматривает как основу для объяснения ситуации выбора. Одновременно она является продуцентом различных убеждений, из которых формируется опыт. Возможны различные варианты моделей-представлений в зависимости от того, какие факторы и свойства субъект принимает во внимание, то есть можно определить множество вариантов представлений $X$, которые будем называть вариантами представлений о ситуации выбора. 
Оценки удовлетворенности или неудовлетворенности текущей ситуацией состояния субъектом, как показано в [13], могут приводить к изменению структуры интересов субъекта и он ее может выбирать. Поскольку предпочтения субъекта в процессе выбора отражают его интересы, можно определить множество $G$ альтернативных вариантов структуры предпочтений, которые будем называть структурными альтернативами $[15,18]$.

Разные типы удовлетворенности или неудовлетворенности характеризуются различными чувствами и отношением. Ситуации выбора, в которых неудовлетворенность продуцируется в целеустремленном индивиде, не знающем, что следует предпринять, образуют для этого субъекта проблемную ситуацию, требующую принятия решения. Стремление субъекта покончить с неудовлетворенностью - и есть принятие решения. Оно может быть связано со способами как устранения проблемы, так и ее разрешения и решения. В любом случае в этом процессе важную роль играют опыт, интуиция, размышления и чувства.

\section{Проектирование системы управления искусственными сущностями на основе теории паттернов}

Разработка цифровых продуктов в виде паттернов поведения в системах управления искусственными сущностями, автоматизирующих выполнение задач миссии, предполагает решение, по крайней мере, двух задач.

Во-первых, фундаментальной задачи перевода наблюдаемого, воспринимаемого и понимаемого паттерна поведения субъекта-лидера другим субъектом (выступающим в роли сенсора), интерпретируемого им как модель (первая и вторая позиции восприятия). Описание в этом случае дается в вербальной форме. Во-вторых, задачи подбора понятий для формирования цифрового описания модели паттерна разработчиками, менеджерами проекта, конструкторами, пользователями, так как они используют разные онтологии для описания идей и понятий (третья и четвертая позиции восприятия).

Основные критерии поведения искусственной сущности: оно должно быть понятным и прогнозируемым, эффективным и корректируемым, то есть допускать коррекцию опыта.

\section{Составные элементы паттерна поведения}

Такой подход требует решения ряда проблем: формирование паттернов поведения че- ловека, проверка им их адекватности и полезности в конкретных ситуациях целеустремленного состояния, формирование представлений человека о ситуации целеустремленного состояния.

Важность изучения этих проблем определяется следующими факторами.

- Понимание поведения связано с тем, как люди описывают ситуацию целеустремленного состояния, делают оценки, строят представление о возможных результатах при использовании различных правил выбора, оценивают их эффективность. Знания о том, как и при каких условиях правило поведения усваивается человеком, должны быть положены в основу поведения искусственной автономной сущности, обладающей волей и интеллектом [1, 19-21].

- Исследование процесса формирования паттернов должно быть основано на переменных окружения, потребностях, мотивах, целях, процессах переработки информации человеком. Наработанные в психологии поведения, организационной психологии и т.п. концептуальные модели должны рассматриваться с позиции их формализации при построении моделей формирования представлений о компонентах ситуации целеустремленного состояния, правил принятия решений.

- Использование теоретических результатов объяснения поведения человека на практике привело к появлению тезиса о том, что, хотя человек и действует нерационально, со временем он осваивает оптимальное правило. Применение теории игр для формализации этого процесса базируется на неопределенных утверждениях относительно понятий равновесия, эффективности, адаптивных концепций и т.п. В связи с этим исследование на новой методологической базе, как и насколько успешно люди учатся на своем опыте, становится важным при анализе различных подходов в теории поведения и ее дальнейшего развития.

- Основной способ понимания человеком полезности созданных им паттернов поведения обратная связь. Она является источником информации для оценки качества и эффективности паттернов, оценки полезности положенных в их основу представлений о ситуации целеустремленного состояния, для определения направления их корректировки, а для исследователя - источником знания о том, как переменные задачи и окружения влияют на способ, которым человек обрабатывает информацию и формирует в памяти субъективные представления о ситуации выбора для объяснения форму- 
лирования правил принятия решений, их изучения и использования.

Формальная модель паттерна поведения приведена в работах [16, 22]. Она предполагает разработку общих ментальных моделей для структурирования объема информации, полученного по результатам наблюдения и одновременного его агрегирования (минимизации), а именно:

- восприятия состояний в предметной области;

- мотивации (зачем что-то делать);

- способов действия (чем и как будем воздействовать на среду и на себя);

- процессов в предметной области (модели объектов управления - как это устроено);

- данных (база типовых ситуаций);

- ценности текущих и возможных состояний (почему это нужно);

- целевой и текущих тактических ситуаций и целей [22];

- ценности ситуаций целеустремленного состояния (тактических и стратегических) по ценности и эффективности;

- удовлетворенности ситуацией целеустремленного состояния по ценности и эффективности [1].

- принятия решений с изменяющейся структурой предпочтений.

Эти элементы образуют то, что можно назвать интеллектуальной цифровой машиной для автоматизации выполнения миссии в интересах субъекта-хозяина (рис. 1).
Перечисленные выше модели всегда присутствуют (возможно, неосознанно) при вы полнении миссии человеком, в том числе и у всех участников проекта при разработке искусственной сущности.

Согласование представлений об архитектуре искусственной сущности путем обмена информацией при обсуждении позволяет определить наиболее эффективную модель паттерна поведения и всех ее составляющих для реализации в искусственной сущности.

С полученной таким образом цифровой машиной положено проводить эксперименты, используя пространственную и визуальную логику для интерпретации результатов, как в методе ТОТЕ (набор входных данных $\rightarrow$ воздействие $\rightarrow$ результат) в смысле Миллера, Галантера, Прибрама [23], в том числе для интерпретации выполнения субпаттернов при изменении субситуаций миссии. Так как пространственное и образное мышление - это способности не вербальные, построение на основе согласования модели легко воспринимается всеми участниками проекта с различными профессиональными онтологиями.

Логика интерпретации базируется на следующих предположениях:

- выбор субъектом осуществляется на основе представлений о ситуации целеустремленного состояния, которая формально определена в [1];

- компоненты представления отражают различные аспекты понимания субъектом си-

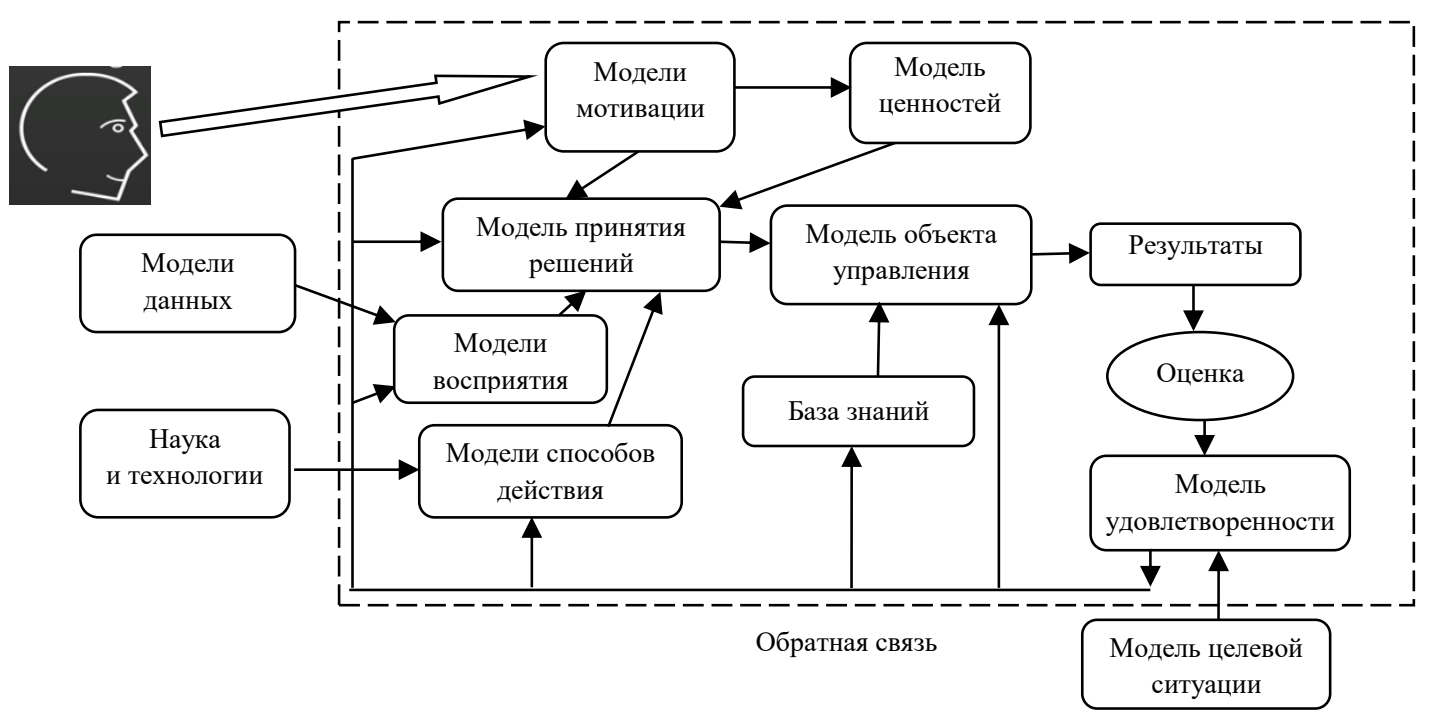

Рис. 1. Архитектура интеллектуальной циифровой машины

Fig. 1. The architecture of the "Intelligent Digital Machine» 
туации целеустремленного состояния и образуют информационную структуру представлений; множество возможных вариантов представлений обозначим через $X$;

- для множества состояний окружения $S$ множество наблюдаемых состояний окружения удовлетворяют условию $S \cap X \neq \varnothing$, то есть представления субъекта могут содержать как объективную составляющую, так и фантомную;

- структурные альтернативы субъект выбирает в зависимости от оценок удовлетворенности значениями свойств ситуации целеустремленного состояния;

- формирование представлений осуществляется на основе процедур восприятия, осознания и анализа согласно когнитивным возможностям субъекта.

В соответствии с введенными предположениями субъект при принятии решений использует три множества альтернатив: управляющие $C$ (способы действия), структурные $G$ (интересы, предпочтения) и идентификации $X$. Следовательно, можно предположить существование трех виртуальных сторон, осуществляющих выбор соответствующих альтернатив. Правила выбора таких альтернатив в зависимости от понимания субъектом обстановки и структуры своих интересов будем называть стратегиями.

\section{Модель восприятия состояний в предметной области}

Представления о ситуации выбора основаны на входной информации, которую субъект извлекает из окружения и опыта. Основное предположение состоит в том, что представления не являются точной копией реальности. Люди не могут непосредственно воспринимать процессы, происходящие в реальности, а лишь фиксируют их проявление органами чувств. На основе информации, получаемой через органы чувств с помощью процессов ощущения, восприятия и осознания окружения и самого себя, а также взаимосвязей между этой информацией, опытом и переживаниями о прошлых ситуациях человек составляет описание наблюдаемых процессов на используемом языке описания. Каждый субъект рефлексирует среду, себя и других субъектов индивидуально, интерпретируя все по-своему, переводя в свою субъективную реальность. Как результат - создается субъективная реальность, которую будем называть субъективными представлени- ями. Представления - это форма описания субъектом окружения и себя в нем в форме паттернов. Они играют (если использовать терминологию теории выбора) роль моделей, с помощью которых человек предсказывает и оценивает возможные результаты от выбираемых способов действия. Следовательно, мы реагируем не на реальность как таковую, а на субъективные модели этой реальности.

У каждого из нас есть собственное мировоззрение, основанное на индивидуальном представлении. Именно оно определяет то, как мы интерпретируем окружающий мир, реагируем на него и какое значение придаем собственному поведению и переживаниям. Через этот механизм и можно осуществлять воздействие на формирование представлений, то есть представления следует рассматривать как объект управления. Первоочередная задача стратегий управления представлениями состоит в их расширении, дополнении, что создает большие возможности для выбора.

Первый принцип теории субъективного выбора, согласно приведенным выше рассуждениям, будем называть принципом формирования субъектных представлений через рефлексию. Рефлексия позволяет моделировать реальность, превращая ее в субъективную. Это свойство характерно для систем, наделенных интеллектом и чувствами.

В системном аспекте под рефлексией следует понимать способность систем строить модели себя и других систем и одновременно видеть себя строящей такие модели [5].

\section{Модель пользователя}

Модель пользователя описывает потребителя, использующего искусственную сущность (например дрон). Она моделирует мотивацию, цели, ценности и оценки. Для проекта создания искусственной сущности построение такой модели должно быть отражено в техническом задании. Модели пользователя, созданные при проектировании интерактивных цифровых продуктов, сред, систем и сервисов, рассмотрены в [24].

Например, для командира вертолета, использующего искусственные сущности дроны, должны быть описаны следующие потребности: как использование дронов позволяет с большей эффективностью выполнять боевые задачи; какие имеются способы оценки поведения дрона; как можно оперативно влиять на изменение его поведения; как можно 
влиять на внесение изменений в «мозги» дрона; какова степень доступа к системе управления дроном для внесения корректировок в конструкцию его цифровой машины по результатам мониторинга выполнения миссии.

Цели командира: выполнить боевую задачу; обеспечить требуемый уровень эффективности ее выполнения; поддерживать живучесть системы вертолет-дрон; знать работу такой системы, уметь прогнозировать ее состояние в неопределенной, слабо структурированной среде.

Его проблемы: динамика боя требует быстрого реагирования на изменение ситуации путем выбора наиболее эффективного способа действия в текущей ситуации; невозможно заранее построить достоверно реализуемый план выполнения боевой задачи; идет постоянная борьба с проблемами, порождаемыми противодействием противника.

Получение подобной информации для построения модели пользователя основано на склонности людей к эмпатии. Она определяет процесс «осознанного сопереживания эмоциональному состоянию другого человека без потери ощущения внешнего происхождения этого переживания». Психологи выделяют три вида эмпатии: когнитивная - базирующаяся на интеллектуальных процессах (типизация, сравнение, аналогия и т.п.); поведенческая высшая форма эмпатии как способность чувствовать состояние другого человека, умение вставать на его позицию; эмоциональная - основанная на сочувствии, сопереживании.

Эмпатия позволяет определить единое понимание предпочтений и потребностей пользователя: чего он ждет от цифровой машины (дрона), которая для него разрабатывается. Она формирует единое понимание и критерий (часто бессознательно) для определения ценности добавления или удаления тех или иных функциональностей.

\section{Модель ценности}

Ценность искусственной сущности как продукта определяется набором ее характеристик (параметров). Их значения позволяют пользователю вынести суждение об этом продукте, то есть что искусственная сущность может делать (набор функциональностей), за счет чего она позволит автоматизировать выполнение миссии (боевую задачу), почему целесообразно использовать этот продукт и в чем его польза.

Функциональность, выступающая как фильтр самого высокого уровня, позволяет отнести ис- кусственную сущность к определенной категории. Если соответствующая категория интересует пользователя, он будет искать ответы на следующие вопросы модели ценности.

Формально модель ценности можно представить в виде оценки удельной ценности по результату, а лингвистически в виде высказывания: [Название продукта] - это категория пользовательского опыта, которая принесет мне такую-то пользу (и лучше альтернативных решений по таким-то причинам).

Последняя часть заключена в скобки, так как не всегда можно описать причины простым предложением. Определить эти отличительные особенности очень важно, но зачастую лучший способ донести их до пользователя - через модель взаимодействия.

Парадокс в том, что модель ценности самая простая для понимания и самая сложная для описания. Потребительская ценность существует и для пользователя, и для организации, которая создает условия для пользовательского опыта. Пользователю важно, как искусственная сущность за счет автоматизации, например, снизит затраты по выполнению задачи (удельная ценность по эффективности), а для организации - достижение поставленной тактической и/или стратегической цели.

Так как организация создает условия для роста удельной ценности по эффективности у пользователя, ценность результата для него первична, а ценность для организации носит производный (зависимый) характер. То есть организация получает ценность как вознаграждение за создание ценности у пользователя.

Самое трудное - найти формулировку, которая определяет ценность для пользователя, а не для организации, создающей пользовательский опыт.

Решение этой проблемы предполагает следующее: выполнение мысленной имитации с помощью бизнес-модели, в которой искусственная сущность - лишь одна составляющая общего ценностного предложения организации для пользователя; для создания убедительной модели ценности построение ее общими усилиями, применяя человеко-ориентированные методы согласования в процессе разработки, привлекая людей, похожих на целевого пользователя.

\section{Модель взаимодействия}

Формализация модели взаимодействия строится на результатах работ [25-27]. Выполнение миссии можно представить как процесс реали- 
зации последовательности ситуаций. На каждом этапе меняются состояние цифровой машины и одновременно состояние среды, которое является генератором условий, определяющих последовательность выполнения этапов. Такой подход к моделированию выполнения миссии следует считать эвристикой.

Пусть принятие решения выполняется в несколько циклических этапов и способы действия выбираются на каждом этапе $n=1,2, \ldots$ из множества $C$ в зависимости от представления о состоянии окружения $x \in X$. Это связано с тем, что совместный надсознательный (интуитивный) и сознательный (формальный) анализы состояния окружения позволяют в многократных итерациях принять сначала смутно осознаваемое, а затем все более четко формулируемое и обоснованное решение. При этом существуют ограничения $C_{x} \subseteq C$ на допустимость выбора альтернатив в зависимости от представлений о состоянии окружения $x \in X$. Динамика процессов в окружении субъекта недоступна прямому восприятию, поэтому представления о ней формируются путем применения процедур идентификации, суть которых сводится к выбору варианта представлений в зависимости от наблюдаемого состояния и структуры интересов $g \in G$. При этом существуют и известны ограничения $X_{s} \subseteq X$ на допустимость представлений в качестве альтернатив идентификации в зависимости от наблюдаемых состояний $s \in S$.

Исходя из этих предположений, следуя [18], введем определения стратегий.

Однозначное отображение $\lambda: X \rightarrow C$ такое, что $\lambda(x) \in C_{x}, x \in X$, называется функцией выбора или управления, упорядоченный набор $\left(\lambda_{1}, \ldots, \lambda_{n}\right) \equiv \lambda_{1}^{n}-$ стратегией выбора на горизонте длины $n<\propto, \lim \left\{\lambda_{1}^{n}\right\}=\lambda_{1}^{n} \propto$ при $n \rightarrow \propto-$ стратегией, направленной на достижение локального идеала, определяющего смысл существования субъекта.

Однозначное монотонное отображение $\xi: S \rightarrow X$, такое, что $\xi(s) \in X_{s}, s \in S$, называется функцией идентификации, упорядоченный набор $\left(\xi_{1}, \ldots, \xi_{n}\right) \equiv \xi_{1}^{n}-$ стратегией идентификации на горизонте длины $n<\propto$, последовательность $\left\{\xi_{1}^{n}, n=1,2, \ldots\right\}$ - стратегией идентификации на ограниченном горизонте. Поскольку субъект стремится к формированию полезных представлений, существует $\lim \left\{\xi_{1}^{n}\right\}=\xi^{\propto}$ при $n \rightarrow \propto$.
Так как множества $S$ и $X$ удовлетворяют условию $|S|>|X|$, однозначное отображение $\xi: S \rightarrow X$ порождает разбиение множества $S$ на подмножества $\xi^{-1}(x)=\bigcup\{s \in S: \xi(s)=x\} \subset S$, $x \in X$. Подмножества $\xi^{-1}(x) \subset S, x \in X$, являются связными множествами, то есть любой элемент $s \in \xi^{-1}(x)$ однозначно определяет соответствующее представление $x \in X$. Следовательно, можно говорить, что подмножества $\xi^{-1}(x) \subset S, x \in X, \quad$ образуют классы эквивалентных представлений. Это позволяет для формализации представлений субъекта использовать методы теории мягких вычислений, например так, как описано в [19].

Выбранная в момент $n$ структурная альтернатива $\gamma_{n} \in G$ является структурным выбором на $n$-м шаге принятия решений, упорядоченный набор $\left(\gamma_{n}, \ldots, \gamma_{1}\right) \equiv \gamma_{1}^{n}-$ стратегией структурного выбора на горизонте принятия решений длины $n<\propto$, последовательность $\left\{\gamma_{1}^{n}\right.$, $n=1,2, \ldots\}$ - стратегией структурного выбора на ограниченном горизонте. Поскольку субъект стремится к соответствию своей структуры интересов требованиям принятой им этической системы, существует $\lim \left\{\gamma_{1}^{n}\right\}=\gamma^{\propto}$ при $n \rightarrow \propto$.

Если модель взаимодействия цифровой машины со средой нельзя описать в виде правдоподобного алгоритма на естественном языке, то первый этап не закончен.

На втором этапе синтеза модели взаимодействия ее представляют в виде композиции элементарных структур (действий), реализация которых не вызывает проблем. В этом случае может потребоваться добавление дополнительных операций для более четкого описания поведения уже готового ПО.

Анализ полученной модели должен быть направлен на минимизацию числа используемых элементарных структур при сохранении работоспособности модели.

При проверке модель взаимодействия должна обсуждаться всеми участниками проекта и прежде всего с пользователем для выработки согласованного решения.

\section{Модель принятия решений с изменяющейся структурой предпочтений}

Согласно [2], критерий выбора стратегии управления имеет смысл ожидаемой удельной ценности целеустремленного состояния по результату, формализация которой имеет вид функции полезности $E \varphi^{g}(C \times S \times X)$, зависящей 
от структурной альтернативы $g \in G$ как от параметра. Поскольку процесс управления начинается с некоторой ситуации $x \in X$, критерий $E \varphi_{n}\left(\lambda_{1}^{n} \mid \gamma_{1}^{n}\right)$ также будет зависеть и от ситуации $x \in X$ как от начального условия. Так как при этом множество ситуаций $X$ конечно, критерий $E \varphi_{n}\left(\lambda_{1}^{n} \mid \gamma_{1}^{n}\right)$ будет окончательно представляться вектором в пространстве $R^{X}$ размерности $|X|$. Его компоненты будем записывать в виде $E \varphi_{n}\left(\lambda_{1}^{n} \mid \gamma_{1}^{n}\right)(x), x \in X$. По результату выбора субъект испытывает эмоциональное переживание, поэтому качество стратегии структурного выбора $\gamma_{1}^{n}$ следует описывать в виде критерия, имеющего смысл удовлетворенности результатами выбора. Следовательно, качество стратегии $\gamma_{1}^{n}$ естественно описывать сверткой вектора ожидаемой полезности $E \varphi_{n}\left(\lambda_{n} \mid \gamma_{1}^{n}\right) \in R^{X} \quad$ в некоторый функционал $\mu: R^{X} \rightarrow R^{1}$. Тогда критерий качества стратегии $\gamma_{1}^{n}$ можно записать в виде $\mu_{n}\left(\lambda_{1}^{n} \mid \gamma_{1}^{n}\right)=$ $=\mu\left(E \varphi_{n}\left(\lambda_{1}^{n} \mid \gamma_{1}^{n}\right)\right) \in R^{1} . \mathbb{F}$

Качество своих представлений субъект связывает с оценками возможности достижения желаемых состояний от управления $c \in C$, а также с возможностью расширения множества $C \uparrow$ путем включения в него эффективных альтернатив. В работе [13] в качестве критерия оценки представлений использованы термы лингвистической переменной «полезность», которые строятся на значениях $E \varphi_{n}\left(\lambda_{1}^{n} \mid \gamma_{1}^{n}\right)$. При этом оценки полезности будут зависеть от стратегий управления $\lambda_{1}^{n}$, структурного выбора $\gamma_{1}^{n}$ как от заданных условий. Обозначим критерий «полезность» $\psi_{n}\left(\xi_{1}^{n} \mid \lambda_{1}^{n}, \gamma_{1}^{n}\right)$. Поскольку процесс идентификации начинается с некоторого состояния $s \in S$, этот критерий будет зависеть от состояния $s \in S$, задаваемого в качестве начального условия. Так как при этом множество состояний $S$ конечно, критерий идентификации будет представляться вектором $\psi_{n}\left(\xi_{1}^{n} \mid \lambda_{1}^{n}, \gamma_{1}^{n}\right)$ в пространстве $R^{S}$ размерности $|S|$.

В ситуации целеустремленного состояния качество стратегий управления и структурного выбора описывается соответственно критериями $E \varphi_{n}\left(\lambda_{1}^{n} \mid \gamma_{1}^{n}\right) \in R^{X}$ и $\mu_{n}\left(\gamma_{1}^{n} \mid \lambda_{1}^{n}\right) \in R^{1}$, имеющими смысл удельной ценности по результату и удовлетворенности результатами выбора, а качество стратегии идентификации - крите- рием $\psi_{n}\left(\xi_{1}^{n} \mid \lambda_{1}^{n}, \gamma_{1}^{n}\right) \in R^{S}$, имеющим смысл полезности представлений для достижения желаемых состояний. Использование введенных критериев предполагает определение соответствующих информационных структур или моделей, позволяющих выполнить соответствующий выбор.

Будем предполагать существование информационной структуры представлений $I$, которая отражает знания и опыт субъекта о способах действия (управления), своих интересах и предпочтениях, о динамике перехода окружения в различные состояния. Тогда можно предположить, что существует преобразование этой структуры в информационную, обеспечивающую возможность построения критерия удельной ценности $E \varphi_{n}\left(\lambda_{1}^{n} \mid \gamma_{1}^{n}\right)$ и модели предметной области. Такое преобразование будем называть преобразованием удельной ценности, а индуцируемую им информационную структуру - информационной структурой удельной ценности ситуации целеустремленного состояния по результату и обозначать $U=U(I)$.

Аналогично, если существует преобразование структуры $I$ в информационную, обеспечивающую возможность построения критерия идентификации $\psi_{n}\left(\xi_{1}^{n} \mid \lambda_{1}^{n}, \gamma_{1}^{n}\right)$ и модели процедур идентификации, то такое преобразование будем называть преобразованием идентификации и обозначать $R$, а индуцируемую им информационную структуру считать информационной структурой идентификации и обозначать $R=R(I)$.

Представления субъекта о ситуации целеустремленного состояния являются субъективными и качественными, построенными на основе наблюдений и анализа процесса перехода окружения под действием управления $c \in C$ в различные состояния $s \in S$. Обозначим правило такого перехода через $q^{g}(S \mid S \times C)$ из $S \times C$ в $S$. Фактически субъект для оценки ценности возможных результатов использует построенную по результатам стратегии идентификации $\xi_{1}^{n}$ модель $Q^{g}(X \mid X \times C)$ из $X \times C$ в $X$. При ее построении учитываются стратегии управления $\lambda_{1}^{n}$ и структурного выбора $\gamma_{1}^{n}$ либо такими стратегиями он задается. Это означает, что преобразование фактической функции $q^{g}(S \mid S \times C)$ в функцию понимания субъектом процессов в его окружении $Q^{g}(X \mid X \times Y)$ возможно лишь в апостериорном режиме в зависимости от используемых стратегий $\left(\lambda_{1}^{n}, \gamma_{1}^{n}, \xi_{1}^{n}\right)$. Такое 
преобразование и построение критерия ожидаемой удельной ценности $E \varphi_{n}\left(\lambda \mid \gamma_{1}^{n}\right)$ возможно при последовательном формировании информационных структур полезности в зависимости от используемых стратегий. Это условие запишем в виде $U_{n}=U\left(\lambda_{1}^{n}, \gamma_{1}^{n}, \xi_{1}^{n}\right)(I), n=1,2, \ldots$ Поскольку это условие является необходимым для формирования критерия ожидаемой полезности и модели предметной области, его необходимо указывать всякий раз при использовании. Отметим, что критерий $E \varphi_{n}\left(\lambda_{1}^{n} \mid \gamma_{1}^{n}\right)$ неявно зависит от стратегии идентификации $\xi_{1}^{n}$ за счет введения в модель выбора индуцированной структуры $U_{n}$. Как было отмечено выше, критерий $\mu_{n}\left(\gamma_{1}^{n} \mid \lambda_{1}^{n}\right) \in R^{1} \quad$ качества структурного выбора определяется сверткой критерия $E \varphi_{n}\left(\lambda_{1}^{n} \mid \tau_{1}^{n}\right) \in R^{X}$. Общность информационной структуры их формирования позволяет записать:

$$
\left\{\begin{array}{l}
E \varphi_{n}\left(\lambda_{1}^{n} \mid \xi_{1}^{n}\right), \\
\mu_{n}\left(E \varphi_{n}\left(\xi_{1}^{n} \mid \lambda_{1}^{n}\right)\right), \\
U_{n}=U\left(\lambda_{1}^{n}, \gamma_{1}^{n}, \xi_{1}^{n}\right)(I) .
\end{array}\right.
$$

Для построения критерия идентификации требуется использовать некоторую функцию, которая имела бы смысл полезности. Для этого надо построить вербальные оценки на значениях функции полезности $E \varphi^{g}(S \times X \times Y)$. Требуемое преобразование существует и может выполняться в априорном режиме (то есть до выбора решений).

Такое преобразование определяется субъектом относительно нечеткой меры, которая может быть построена, если задана функция $q^{g}(S \mid S \times C)$ из $S \times C$ в $S$. Поскольку ее аналог в сознании субъекта имеет вид $Q^{g}(X \mid X \times C)$ и может однозначно задать ее в информационной структуре $I$, дополнительные преобразования не требуются. Построение функции полезности представлений исчерпывает необходимое структурное преобразование. Такое преобразование будем называть структурным преобразованием идентификации и обозначать $R$, а индуцируемую им информационную структуру информационной структурой полезности представлений и обозначать $R=R(I)$.

С учетом этих соображений критерий идентификации окончательно запишем в виде

$$
\left\{\begin{array}{l}
\psi_{n}\left(\xi_{1}^{n} \mid \lambda_{1}^{n}, \gamma_{1}^{n}\right) \\
R=R(I) .
\end{array}\right.
$$

Из введенных определений и построений следует, что критерии качества этих видов стратегий различны и взаимозависимы. Тогда проблема выбора имеет игровое содержание и сводится к отысканию устойчивого компромисса между стремлением к максимизации ожидаемой удельной ценности целеустремленного состояния по результату и минимизации возможных потерь от неправильных представлений. Такой компромисс называется равновесием.

Поскольку информационная структура удельной ценности $U_{n}=U\left(\lambda_{1}^{n}, \gamma_{1}^{n}, \xi_{1}^{n}\right)(I)$, в условиях которой строится критерий $\mu_{n}\left(E \varphi_{n}\left(\gamma_{1}^{n} \mid \lambda_{1}^{n}\right)\right)$, должна формироваться последовательно в зависимости от используемых стратегий, искомые равновесия будут взаимозависимыми на каждом этапе $n=1,2, \ldots$ формирования решений, а также зависимыми от решений, выбираемых на предшествующих шагах. С учетом этого естественно называть равновесия динамическими.

Три стратегии $\left\{\lambda_{1}^{o_{n}}, \gamma_{1}^{o_{n}}, \xi_{1}^{o_{n}}\right\}$, удовлетворяющие условиям

$$
\left\{\begin{array}{l}
E \varphi_{n}\left(\lambda_{1}^{o_{n}} \mid \gamma_{1}^{o_{n}}\right) \geq E \varphi_{n}\left(\lambda_{1}^{n} \mid \gamma_{1}^{o_{n}}\right) \quad \forall \lambda_{1}^{n}, \\
\mu_{n}\left(\gamma_{1}^{o_{n}} \mid \lambda_{1}^{o_{n}}\right) \geq \mu_{n}\left(\gamma_{1}^{n} \mid \lambda_{1}^{o_{n}}\right) \quad \forall \gamma_{1}^{n}, \\
U_{n}=U\left(\lambda_{1}^{o_{n}}, \gamma_{1}^{o_{n}}, \xi \gamma_{1}^{o_{n}}\right)(I)
\end{array}\right.
$$

и

$$
\left\{\begin{array}{l}
\psi_{n}\left(\xi_{1}^{o_{n}} \mid \gamma_{1}^{o_{n}}, \lambda_{1}^{o_{n}}\right) \geq \mu_{n}\left(\xi_{1}^{o_{n}} \mid \gamma_{1}^{o_{n}}, \lambda_{1}^{o_{n}}\right) \quad \forall \xi_{1}^{n}, \\
R=R(I), n=1,2, \ldots
\end{array}\right.
$$

называются динамическими равновесиями (см. работы $[13,18])$.

Согласно предположениям, количество циклов формирования решения неограниченно. Тогда динамические равновесия должны иметь смысл, в том числе при $n \rightarrow \propto$.

Для его существования естественно потребовать выполнения следующих дополнительных условий: при $n \rightarrow \propto$ критерии качества стратегий должны стремиться к некоторым пределам, такие пределы не должны зависеть от начальных условий.

Поскольку критерии в явном виде не заданы, выполнение этих свойств не очевидно. Это требует задания нужных свойств и затем указания явного вида критериев, удовлетворяющих этим свойствам.

Согласно введенным предположениям, критерии качества стационарных стратегий $\lambda^{n}, \gamma^{n}, \xi^{n}$ при $n \rightarrow \propto$ имеют пределы, тогда три стационарные стратегии $\left(\lambda^{o_{\infty}}, \gamma^{o_{\infty}}, \xi^{o_{\infty}}\right)$ называются стационарными равновесиями, если для 
них существуют пределы, удовлетворяющие условиям:

$$
\left\{\begin{array}{l}
\left\{\begin{array}{l}
\varphi\left(\lambda^{o_{\infty}} \mid \gamma^{o_{\infty}}\right) \geq \varphi_{n}\left(\lambda^{\infty} \mid \gamma^{o_{\infty}}\right), \forall \lambda^{\infty} \\
\mu\left(\gamma^{o_{\infty}} \mid \lambda^{o_{\infty}}\right) \geq \mu_{n}\left(\gamma^{\infty} \mid \lambda^{o_{\infty}}\right), \forall \gamma^{\infty} \\
U=U\left(\lambda^{o_{\infty}}, \gamma^{o_{\infty}}, \xi^{o_{\infty}}\right)(I)
\end{array}\right. \\
\left\{\begin{array}{l}
\psi\left(\xi^{o_{\infty}} \mid \lambda^{o_{\infty}}, \gamma^{o_{\infty}}\right) \geq \psi\left(\xi^{\infty} \mid \lambda^{o_{\infty}} \gamma^{o_{\infty}}, \forall \xi^{\infty},\right. \\
R=R(I)
\end{array}\right.
\end{array}\right.
$$

Таким образом, содержание проблемы моделирования выбора состоит в отыскании компромисса между стремлением к достижению максимальной ожидаемой удельной ценности по результату и минимальных потерь от неверных представлений с учетом их взаимной зависимости. Согласно принципу равновесных решений, он не должен улучшаться одновременно по всем компонентам интересов.

При достижении такого компромисса можно утверждать, что интересы субъекта реализуются с наилучшим результатом. Если при этом динамические равновесия удовлетворяют требованиям асимптотической стационарности, то можно также утверждать, что интересы субъекта реализуются с наилучшим результатом на всем бесконечном горизонте, в том числе при $n \rightarrow \propto$. Отсюда следует, что динамические равновесия определяют смысл и способ реализации интересов с наилучшим результатом. С учетом этого динамические равновесия естественным образом определяют внутреннюю цель при принятии решений.

\section{Пример}

Пусть требуется создать робота-велосипедиста для гонок [28]. Понятно, что в этом случае нужно выполнить определенное абстрагирование, то есть выделить только те элементы системы, которые участвуют во взаимодействии велосипедиста с дорогой (рис. 2).

Будем выполнять такое абстрагирование в виде паттерна и в описании выявлять значимые для анализа существительные.

При езде велосипедист сидит на сиденье, поместив ноги на педали, руками держит руль. Он управляет велосипедом, контролируя угол наклона и поворот руля в определенном диапазоне так, чтобы не упасть под действием силы тяжести и добраться из исходного пункта в место назначения, то есть совершить поездку.

Как видим, словесное описание алгоритма представляет собой схематичный набор правил, или план, состоящий из различных дей-

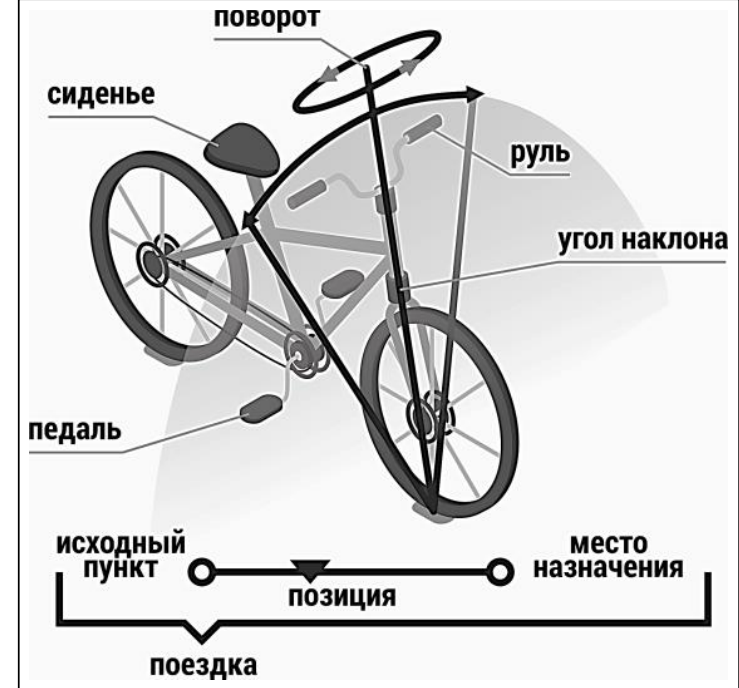

Рис. 2. Симулятор езды на велосипеде с роботом-велосипедистом

Fig. 2. Bike riding simulator with a robot cyclist

ствий, реализация которых приводит к желаемому результату. По сути действия в виде ключевых слов - это процедуры, имеющие свой алгоритм реализации с точками обмена информацией с другими процедурами и средой. Применение принципа последовательной детализации и использование типовых управляющих структур, структур данных, встроенных объектов, методов и событий позволяют разрабатывать автоматизированный вариант сформулированного набора правил.

Используя методы проектирования алгоритмов на псевдокоде, это можно представить следующим образом:

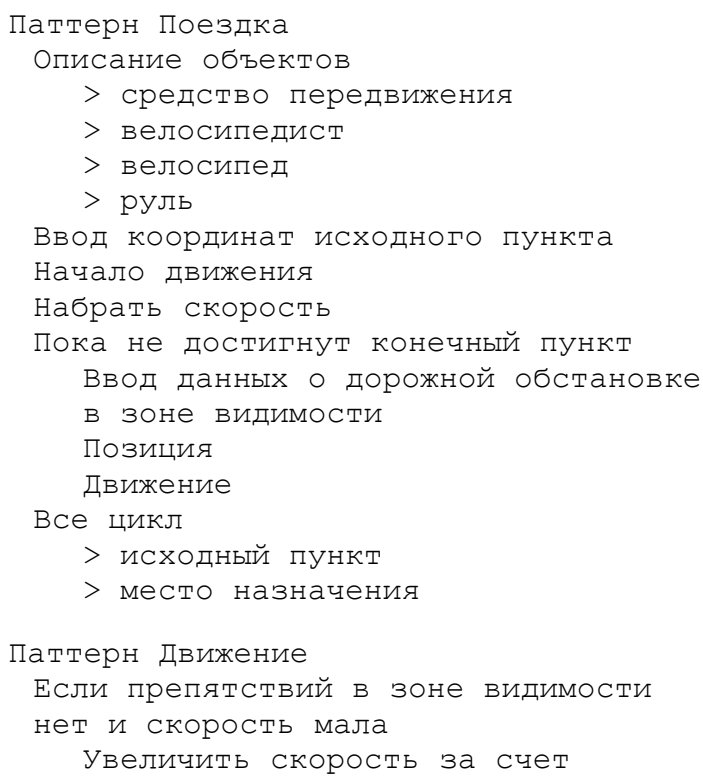


увеличения частоты вращения педалей Если расстояние до препятствия

большое и скорость движения большая

Продолжить движение, постепенно уменьшить скорость до средней

Если расстояние до препятствия среднее

и скорость движения большая или средняя Уменьшить скорость до малой

Если расстояние до препятствия малое

Погасить скорость, объехать

препятствие

Если препятствий в зоне видимости

нет и скорость большая и дорога прямая

Продолжить движение в данном

направлении с той же скоростью

Если препятствий в зоне видимости

нет и виден поворот дороги

Выполнить поворот

Конец

Паттерн объехать

Выбрать сторону объезда

$>$ поворот

$>$ позиция

Конец

Паттерн Поворот

$>$ угол наклона

Паттерн Торможение

Здесь каждое слово - это наименование процедуры, которая описывает элементарное действие (понятие). Такое представление как выявляет объекты в модели, так и определяет их взаимодействие. Иерархическая структура, которая выявлена в модели взаимодействия, естественным образом появляется и здесь, поскольку эти две модели взаимосвязаны. Чем подробнее рассмотрены паттерны в модели взаимодействия, тем выше детализация и точность описания объектов, вступающих во взаимоотношения. Верно и обратное: если разбить объекты на подобъекты, история их взаимодействия станет более подробной.

Этот алгоритм существенно зависит от механизма сбора и интерпретации данных о состоянии обстановки.

\section{Модель данных}

Будем считать, что агент способен выделять факторы - характеристики окружения $X^{k}=$ $=\left\{x_{i}^{k}, i=\overline{1, N}\right\}$. Влияние каждого фактора агент оценивает с помощью лингвистической переменной «степень влияния фактора»: $\mu_{x}^{k}\left(x_{i}^{k}\right): x_{i}^{k} \rightarrow$ $\rightarrow[0,1]$. Введем параметр, с помощью которого агент оценивает свою ситуационную осведомленность в ситуации целеустремленного состояния:

$$
E s^{k}=\frac{\sum_{i=1}^{N} \mu_{x}^{k}\left(x_{i}^{k}\right) x_{i}^{k}}{\sum_{i=1}^{N} \mu_{x}^{k}\left(x_{i}^{k}\right)} .
$$

Можно определить следующее ограничение: $\sigma^{k}\left(E s^{k}\right) \geq \sigma_{0}^{k}$, где $\sigma_{0}^{k}-$ некоторый пороговый уровень осведомленности агента от использования собственных источников информации.

Модель данных строится на основе использования понятия лингвистической переменной, которая имеет имя и набор термов. Это означает, что при представлении данных от сенсоров они преобразуются в лингвистические значения. Таким образом, фиксируются данные о состоянии и определяются свойства объекта. Фиксация состояния всех объектов в машине по отдельности позволяет определить состояние машины в целом.

Такой подход позволяет управлять скоростью и направлением движения велосипеда голосом: числовые данные, получаемые от моделей сенсоров, переводятся в лингвистические значения и используются в модели (1), затем расчетные значения изменения состояния переводятся в визуальную информацию для получения сигнала обратной связи. Визуализацию целесообразно выполнять на карте обстановки реального объекта, например, трехмерной карте города.

Таким образом, вся работа по разработке и реализации, выполняемая участниками проекта, сводится к тому, чтобы дать пользователю возможность изменять несколько лингвистических параметров. Понимание этого проясняет ситуацию и упрощает формирование согласованного представления о возможностях цифровой машины.

\section{Заключение}

Формальная модель паттерна поведения субъекта-лидера позволила сформировать архитектуру искусственной сущности как некоторой цифровой машины, представленной в работе как совокупность десяти моделей. Показано, что эти модели в той или иной форме, в том числе и неосознанно, присутствуют и у разработчиков, и у потребителей этой машины.

Достичь желаемого резкого повышения эффективности подобных систем и комплексов возможно, главным образом, путем организации по определенным правилам коммуникации разработчиков отдельных элементов модели 
паттерна. Это позволяет выявить неосознанные предположения и допущения на ранней стадии и оценить степень согласованности решений, предложенных участниками проекта. Использование модели заказчика образует так называ- емый корпоративный интеллект, что позволяет обеспечить проектирование в соответствии с функциональным назначением искусственной сущности в неопределенной и плохо формализуемой среде.

\section{Лuтература}

1. Виноградов Г.П. Моделирование принятия решений интеллектуальным агентом // Программные продукты и системы. 2010. № 3. С. 45-51.

2. Городецкий В.И., Самойлов В.В., Троцкий Д.В. Базовая онтология коллективного поведения автономных агентов и ее расширения // Изв. РАН. Теория и системы управления. 2015. № 5. С. $102-121$. DOI: $10.7868 / \mathrm{S} 0002338815030087$.

3. Новиков Д.А., Чхартишвили А.Г. Прикладные модели информационного управления. М.: Издво ИПУ РАН, 2004. 129 с.

4. Новиков Д.А., Чхартишвили А.Г. Рефлексивные игры. М.: Синтег, 2003. 149 с.

5. Лефевр В.А. Формула человека: Контуры фундаментальной психологии; [пер. с англ.]. М.: Прогресс, 1991. 108 с. $255 \mathrm{c}$.

6. Лепский В.Е. Рефлексивно-активные среды инновационного развития. М.: Когито-Центр, 2010.

7. Foundation for Intelligent Physical Agents. FIPA ACL Message Structure Specification. URL: http://fipa.org/specs/fipa00061/SC00061G.pdf (дата обращения: 19.01.2021).

8. Bonovi F., Milito R., Zhu J., Addepalli S. Fog computing and its role in the internet of things. Proc. MCC'12, 2012, pp. 13-16. DOI: 10.1145/2342509.2342513.

9. Городецкий В.И. Самоорганизация и многоагентные системы. І. Модели многоагентной самоорганизации // Изв. РАН. Теория и системы управления. 2012. № 2. С. 92-120.

10. Городецкий В.И. Самоорганизация и многоагентные системы. ІІ. Приложения и технология разработки // Изв. РАН. Теория и системы управления. 2012. № 3. С. 102-123.

11. Вентцель Е.С. Исследование операций. Задачи, принципы, методология. М.: Наука, 1988. 208 с.

12. Акофф Р.Л., Эмери Ф.Е. О целеустремленных системах; [пер. с англ.]. М.: Сов. радио, 1974. $272 \mathrm{c}$.

13. Виноградов Г.П., Кирсанова Н.В., Фомина Е.Е. Теоретико-игровая модель целенаправленного субъективно рационального выбора // Нейроинформатика. 2017. Т. 10. № 1. С. 1-12.

14. Vinogradov G.P. A subjectve rational choice. JPCS, 2017, vol. 803, art. 012176. DOI: 10.1088/ 1742-6596/803/1/012176.

15. Бурков В.Н., Виноградов Г.П., Кузнецов В.Н., Палюх Б.В., Семенов Н.А. Интеллектуальные активные системы // КИИ: тр. конф. 2010. Т. 3. С. 35-43.

16. Виноградов Г.П., Прохоров А.А., Шепелев Г.А. Паттерны в системах управления автономными системами // Информационные и математические технологии в науке и управлении. 2020. № 1. С. 4054. DOI: 10.38028/ESI.2020.17.1.003.

17. Лефевр В.А. Конфликтующие структуры. М.: Сов. радио, 1973.158 с.

18. Баранов В.В. Динамические равновесия в задачах стохастического управления и принятия решений при неопределенностях // Изв. РАН. Теория и системы управления. 2002. № 3. С. 77-93.

19. Виноградов Г.П., Борисов П.А., Семенов Н.А. Интеграция нейросетевых алгоритмов, моделей нелинейной динамики и методов нечеткой логики в задачах прогнозирования // Изв. РАН. Теория и системы управления. 2008. № 1. С. 78-84.

20. Виноградов Г.П., Шматов Г.П., Борзов Д.А. Формирование представлений агента о предметной области в ситуации выбора // Программные продукты и системы. 2015. № 2. С. 83-94. DOI: 10.15827/ 0236-235X.110.083-094.

21. Edwards W., Tversky A. Decision Making. Harmoudsworth: Penguin Publ., 1967, 412 p.

22. Виноградов Г.П. Системы управления на основе паттернов // Software Journal: Theory and Applications. 2020. № 2. C. 10-19. URL: http://swsys-web.ru/en/pattern-control-system.html (дата обращения: 16.01.2021).

23. Миллер Дж., Галантер Е., Прибрам К. Планы и структура поведения; [пер. с англ.]. М.: Прогресс, 1964. $238 \mathrm{c}$.

24. Johnson J., Henderson A. Conceptual models: core to good design. Synthesis Lectures on Human-Centered Informatics, 2011, vol. 4, no. 2, pp. 1-110. DOI: 10.2200/S00391ED1V01Y201111HCI012. 
25. Hornbaek K., Oulasvirta A. What interaction. Proc. CHI, 2017, pp. 5040-5053. DOI: 10.1145/3025453.302 5765. $651 \mathrm{p}$.

26. Cooper A., Reimann R., Cronin D. About Face 3: The Essentials of Interface Design. Wiley Publ., 2007,

27. Дилтс Р. Изменение убеждений с помощью НЛП; [пер. с англ.]. М.: Независимая фирма «Класс», 1997. $192 \mathrm{c}$.

28. Разработка цифровых продуктов с помощью ментальных моделей. URL: https://sobakapav.ru/ publications/designing-digital-mental-models (дата обращения: 16.01.2021).

\section{Mental models in designing the behavior of artificial objects}

Vinogradov G.P. ${ }^{1,2}$, Dr.Sc. (Engineering), Professor, Head of Laboratory, wgp272ng @mail.ru

${ }^{1}$ Tver State Technical University, Tver, 170026, Russian Federation

${ }^{2} R \& D$ Institute Centerprogramsystem, Tver, 170024, Russian Federation

Abstract. The authors relate the immediacy of the problem considered in this paper to the need to design artificial systems to perform a certain mission like a person and when interacting with him.

The analysis of approaches to the construction of artificial objects has shown that developers often give artificial objects their own patterns of behavior because of the specifics of the concepts and concepts they use. As a result, there are gaps between the formal models of patterns offered by developers and the expectations of users.

The aim of the authors is to justify an approach to the development of software for intelligent systems for managing the behavior of artificial entities based on the theory of patterns and to propose an approach that provides the development of digital products based on the patterns of behavior of the subject-leader for artificial entity management systems that automate the implementation of mission problems.

The work uses the methods of the theories of reflexive games and information management of systems with willpower and intelligence. There are ten constituent elements based on a formal model of the behavior pattern. The work shows that these elements form what can be called an intelligent digital machine designed to automate the execution of a mission in the interests of the host subject. These components are present when a person performs a mission, including all participants in the project to develop an artificial entity. The authors show that the ideas adjustment about the architecture of an artificial entity through the exchange of information during the discussion allows us to determine the most effective model of the behavior pattern and all its components for implementation in an artificial entity. The authors proposed to conduct matching as a game by conducting experiments using the TOTHE method (a set of input data $\rightarrow$ impact $\rightarrow$ result), using spatial and visual logic to interpret the results.

The possible logic of interpretation is justified. The authors briefly considered the models of the constituent elements of the behavior pattern for an artificial entity. An example of the approach implementation is considered. The authors show that in conditions of severe time scarcity; the choice based on behavior patterns makes it possible to implement effective behavior that does not require significant computational resources.

Keywords: decision making, goal-oriented systems, fuzzy judgment, the pattern, the choice situation.

\section{References}

1. Vinogradov G.P. Model of decision-making by the intellectual agent. Software and Systems, 2010, no. 3, pp. $45-51$ (in Russ.).

2. Gorodetsky V.I., Samoylov V.V., Trotsckii D.V. The reference ontology of collective behavior of autonomous agents and its extensions. J. Comput. Syst. Sci. Int., 2015, vol. 54, no. 5, pp. 765-782. DOI: $10.1134 /$ S1064230715030089.

3. Novikov D.A., Chhartishvili A.G. Applied Models of Information Management. Moscow, 2004, 129 p. (in Russ.).

4. Novikov D.A., Chhartishvili A.G. Reflective Games. Moscow, 2003, 149 p. (in Russ.).

5. Lefebvre V.A. The Formula of Man an Outline of Fundamental Psychology. 1991. (Russ. ed.: Moscow, 1991, 108 p.). 
6. Lepsky V.E. Reflexive-Active Environments of Innovative Development. Moscow, 2010, 255 p. (in Russ.).

7. Foundation for Intelligent Physical Agents. FIPA ACL Message Structure Specification. Available at: http://fipa.org/specs/fipa00061/SC00061G.pdf (accessed January 19, 2021).

8. Bonovi F., Milito R., Zhu J., Addepalli S. Fog computing and its role in the internet of things. Proc. MCC'12, 2012, pp. 13-16. DOI: 10.1145/2342509.2342513.

9. Gorodetsky V.I. Self-organization and multiagent systems. I. Models of multiagent self-organization. J. Comput. Syst. Sci. Int., 2012, vol. 51, no. 2, pp. 256-281. DOI: 10.1134/S106423071201008X.

10. Gorodetsky V.I. Self-organization and multiagent systems. II. Applications and the development technology. J. Comput. Syst. Sci. Int., 2012, vol. 51, no. 3, pp. 391-409. DOI: 10.1134/S1064230712020062.

Russ.).

11. Ventcel E.S. Operations Research. Objectives, Principles, Methodology. Moscow, 1988, 208 p. (in

12. Ackof R.L., Emery F.E. On Purposeful Systems. 1972, 288 p. (Russ. ed.: Moscow, 1974, 272 p.).

13. Vinogradov G.P., Kirsanova N.V., Fomina E.E. Game-theoretic model of targeted subjectively rational choice. Neiroinformatics, 2017, vol. 10, no. 1, pp. 1-12 (in Russ.).

14. Vinogradov G.P. A subjectve rational choice. JPCS, 2017, vol. 803, art. 012176. DOI: 10.1088/17426596/803/1/012176.

15. Burkov V.N., Vinogradov G.P., Kuznetsov V.N., Palyuh B.V., Semenov N.A. Intelligent active systems. Proc. RCAI, 2010, vol. 3, pp. 35-43 (in Russ.).

16. Vinogradov G.P., Prokhorov A.A., Shepelev G.A. Patterns in control systems by autonomous systems. Informational and Mathematical Technologies in Science and Management, 2020, no. 1, pp. 40-54. DOI: 10.38028/ESI.2020.17.1.003 (in Russ.).

17. Lefebvre V.A. Conflicting Structures. Moscow, 1973, 158 p. (in Russ.).

18. Baranov V.V. Dynamic equilibria in problems of stochastic control and decision making under uncertainty. J. Comput. Syst. Sci. Int., 2002, vol. 41, no. 3, pp. 409-425.

19. Vinogradov G.P., Borisov P.A., Semenov N.A. Integration of neural network algorithms, nonlinear dynamics models, and fuzzy logic methods in prediction problems. J. Comput. Syst. Sci. Int., 2008, vol. 47, no. 1 , pp. $72-77$.

20. Vinogradov G.P., Shmatov G.P., Borzov D.A. Formation of agent's representations of the domain in a situation of choice. Software and Systems, 2015, no. 2, pp. 83-94. DOI: 10.15827/0236-235X.110.083-094 (in Russ.).

21. Edwards W., Tversky A. Decision Making. Harmoudsworth: Penguin Publ., 1967, 412 p.

22. Vinogradov G.P. Pattern control system. Software Journal: Theory and Applications, 2020, no. 2, pp. 10-19. Available at: http://swsys-web.ru/en/pattern-control-system.html (accessed January 16, 2021).

23. Miller G.A., Galanter G., Pribram K.H. Plans and the Structure of Behaviour. NY, 1960. DOI: 10.1037/10039-000. (Russ. ed.: Moscow, 1964, 238 p.).

24. Johnson J., Henderson A. Conceptual models: core to good design. Synthesis Lectures on Human-Centered Informatics, 2011, vol. 4, no. 2, pp. 1-110. DOI: 10.2200/S00391ED1 V01Y201111HCI012.

25. Hornbaek K., Oulasvirta A. What interaction. Proc. CHI, 2017, pp. 5040-5053. DOI: 10.1145/3025453. 3025765. $651 \mathrm{p}$.

26. Cooper A., Reimann R., Cronin D. About Face 3: The Essentials of Interface Design. Wiley Publ., 2007,

27. Dilts R. Changing Belief Systems with NLP. Meta Publ., 1990, 221 p. (Russ. ed.: Moscow, 1997, 192 p.).

28. Sheiner T. Designing Digital Products with Mental Models. Available at: https://medium.com/ salesforce-ux/designing-digital-products-with-mental-models-45ac5c0a9dc2 (accessed January 16, 2021).

\section{Для цитирования}

Виноградов Г.П. Ментальные модели в проектировании поведения искусственных сущностей // Программные продукты и системы. 2021. T. 34. № 1. C. 019-032. DOI: 10.15827/0236235X.133.019-032.

\section{For citation}

Vinogradov G.P. Mental models in designing the behavior of artificial objects. Software \& Systems, 2021, vol. 34, no. 1, pp. 019-032 (in Russ.). DOI: 10.15827/0236-235X.133.019-032. 